\title{
An apparent quantized thermal Hall effect in a magnetic insulator
}

\author{
Majorana quantization and half-integer thermal quantum Hall effect in a \\ Kitaev spin liquid \\ Authors: Y. Kasahara, T. Ohnishi, Y. Mizukami, O. Tanaka, Sixiao Ma, K. Sugii, N. \\ Kurita, H. Tanaka, J. Nasu, Y. Motome, T. Shibauchi, Y. Matsuda \\ arXiv:1805.05022
}

\section{Recommended with a Commentary by Leon Balents, Kavli Institute of Theoretical Physics, UCSB}

Physicists are fascinated by emergent excitations, which arise in many-body quantum systems and behave like particles with quantum numbers not found amongst the elementary particles of nature. Such quasi-particles can exhibit fractional charges, and, more fundamentally, fractional statistics. Examples found in experiment have been infrequent but impactful: solitons in polyacetylene, spin-1/2 spinons in one dimensional spin chains, and Laughlin quasi-particles in the fractional quantum Hall effect in two dimensional electron gases. Laughlin quasi-particles are known to possess fractional charge and fractional statistics, and as a consequence of the latter, they are considered "anyons". The fractional charge has been demonstrated experimentally in shot noise and other experiments, and while it is widely believed that the fractional statistics assumption is correct, the experimental verification of those statistics is still controversial. Since the discovery of the fractional quantum Hall effect, there has been a notable pause in the progression of such experimental findings.

Theory, however, continues to develop new ground, and has proposed both many new types of emergent quasi-particles and new venues for them. Starting with work of Wen, Moore and Read in the 1990s, a new type of anyon with not just fractional but non-abelian statistics was proposed to exist in the fractional quantum Hall regime (the idea appeared more abstractly in the field theory literature somewhat earlier). A system of non-abelian anyons is accompanied by an internal Hilbert space, whose dimension grows with the number of anyons, and braiding of anyons enacts unitary transformations upon this space. The concept of topological quantum computing is based on this structure. Separately from the quantum Hall work, a long thread of study initiated by Phil Anderson in the 1970s has proposed states dubbed quantum spin liquids to exist in quantum magnets with low spin and frustrated interactions. They are predicted to host spin-1/2 neutral spinons (as well as other exotic quasi-particles) in two and three dimensions.

The simplest non-abelian anyon is a particle carrying a Majorana fermion zero mode, which theory predicts may occur in certain topological phases of matter. The latter include spin-polarized $\mathrm{p}+\mathrm{ip}$ superconductors, a topological insulator surface proximity coupled to a 
superconductor, and certain composite structures combining semiconductors and superconductors. In two dimensions, all these examples are described by the topological field theory of Ising anyons. Another example of Ising anyons was introduced by Alexei Kitaev - who originated many of the above ideas - in his soluble model of spin- $1 / 2$ spins on a honeycomb lattice[1]. This system is a quantum spin liquid, and the Ising anyon state occurs when a magnetic field is applied along certain directions. In Kitaev's honeycomb model, Majorana fermions are gapless in the bulk at zero field, but become gapped in a field, producing the non-abelian topological phase. This example differs from the former superconducting ones in that there are no microscopic fermions in the spin system, and there is no $\mathrm{U}(1)$ charge quantum number, but the topological content is the same.

In addition to the bulk Ising anyons, the topological spin liquid state, like the $\mathrm{p}+\mathrm{ip}$ superconductor, possesses a chiral Majorana fermion edge state, whose Hamiltonian is

$$
H=\frac{-i \hbar v}{4} \int d x \eta \partial_{x} \eta=\sum_{k>0} \hbar v k \eta_{k}^{\dagger} \eta_{k},
$$

where $\left\{\eta(x), \eta\left(x^{\prime}\right)\right\}=2 \delta\left(x-x^{\prime}\right)$, and $\left\{\eta_{k}, \eta_{k^{\prime}}^{\dagger}\right\}=\delta_{k, k^{\prime}}$ are canonical fermion operators, and $v$ is the edge velocity. A classroom level calculation gives the heat current carried by this edge state (if it is) at a constant temperature $T$ as

$$
I_{Q}=\sum_{k>0} v \times \hbar v k \times \frac{f(\hbar v k)}{L}=c \frac{\pi}{12} \frac{k_{B}^{2} T^{2}}{\hbar},
$$

where $f$ is the Fermi function, and $c=1 / 2$ is the chiral central charge of a Majorana fermion. Considering a Hall bar with top and bottom edges with opposite propagation direction and a small temperature difference $\Delta T$, and assuming no current in the bulk, one obtains by subtraction a quantized thermal Hall conductance

$$
\frac{K_{x y}}{T}=c \frac{\pi}{6} \frac{k_{B}^{2}}{\hbar} .
$$

This conductance is equal to the corresponding conductivity in two dimensions. The same expression holds for the thermal conductivity/conductance of quantum Hall states, but with $c$ taking integer values, even for the Laughlin series of fractional quantum Hall states. Thus a fractional value of $c$ is a strong indicator of physics beyond even those phases, and to my knowledge quantization with $c<1$ implies non-abelian anyons. However, even in the quantum Hall effect, the thermal conductance of edge channels has only recently been measured[2, 3]. See the articles and commentary for December 2017 here.

The highlighted paper reports measurements of the thermal Hall conductivity with an applied magnetic field in $\alpha-\mathrm{RuCl}_{3}$, an insulating magnetic material with a two-dimensional honeycomb lattice. It is an example of a so-called "Kitaev material" [4], in which has been argued that localized effective $\mathrm{S}=1 / 2$ spins (here from $\mathrm{Ru}^{3+}$ ions) experience strong interactions of the type in Kitaev's soluble honeycomb model. In $\alpha-\mathrm{RuCl}_{3}$, various indications suggest the Kitaev exchange interaction is $50-90 \mathrm{~K}$, while at zero field the system orders antiferromagnetically at about $7 \mathrm{~K}$. The disparity between these energies has been interpreted by many researchers as evidence for proximity to Kitaev's quantum spin liquid state. Many 
experimental techniques have been brought to bear on this system, but the presence of Majorana fermions or indeed of any quantum spin liquid state cannot be unequivocally deduced from these observations.

A magnetic field destroys the antiferromagnetic order in $\alpha-\mathrm{RuCl}_{3}$ when the in-plane component of the field exceeds about 6.5T. The measured $K_{x y}$ presented in the highlighted paper, obtained for a field at an angle of 30 degrees to the plane, exhibits a plateau at the quantized value with $c=1 / 2$ when the in-plane field is between about 7-9T, for temperatures between $2-5 \mathrm{~K}$. While this is a first observation and not yet reproduced by any other groups, if confirmed, a quantized thermal Hall conductivity would be very difficult to explain without a chiral Majorana edge state. If indeed this reflects Majorana fermions, then it could be a landmark discovery, indicating a non-abelian Ising anyon phase at zero temperature in this field range. One interesting question raised by the experiment is the role of the lattice: in the experiments the longitudinal thermal conductivity, $\kappa_{x x}$, is approximately a thousand times larger than the Hall component. In the electrical Hall effect, quantization fails when $\sigma_{x x} \gg \sigma_{x y}$. The highlighted paper attributes the large $\kappa_{x x}$ to phonons, which provide a parallel conduction channel for heat that potentially might not disrupt the quantization of $\kappa_{x y}$. However, as discussed in two recent preprints [5, 6], the persistence of quantization under these conditions is subtle, and it will be interesting to see this explored experimentally in the future.

\section{References}

[1] Alexei Kitaev. Anyons in an exactly solved model and beyond. Annals of Physics, 321(1):2-111, 2006.

[2] Mitali Banerjee, Moty Heiblum, Vladimir Umansky, Dima E Feldman, Yuval Oreg, and Ady Stern. Observation of half-integer thermal hall conductance. Nature, page 1, 2018.

[3] Mitali Banerjee, Moty Heiblum, Amir Rosenblatt, Yuval Oreg, Dima E Feldman, Ady Stern, and Vladimir Umansky. Observed quantization of anyonic heat flow. Nature, 545(7652):75, 2017.

[4] Simon Trebst. Kitaev materials. arXiv preprint arXiv:1701.07056, 2017.

[5] Mengxing Ye, Gábor B Halász, Lucile Savary, and Leon Balents. Quantization of the thermal hall conductivity at small hall angles. arXiv preprint arXiv:1805.10532, 2018.

[6] Yuval Vinkler-Aviv and Achim Rosch. Approximately quantized thermal hall effect of chiral liquids coupled to phonons. arXiv preprint arXiv:1805.11587, 2018. 